\title{
Research Multi-Sensor Data Fusion Algorithm based on the adaptive cubature kalman filter
}

\author{
Xiuguo Zhang \\ School of Electronics and Information Engineering of Zhu Hai City Polytechnic, Zhuhai, Guangdong, \\ 519090, China
}

\begin{abstract}
Keywords: Cubature kalman filter; adaptive; noise statistic estimator; correction function; integrated filtering; data fusion.
\end{abstract}

\begin{abstract}
Cubature kalman filter is prone to filtering divergence if the system model is inaccurate or measuring abnormal. In order to solve this problem, the adaptive cubature kalman filter algorithm was proposed in this paper, it constructed a group of noise statistic estimators to estimate the statistical characteristic of the noise in real time; and when the measurement anomaly, it adopted correction function to adjust the filtering process, and thus the estimation accuracy and the ability to restrain the filtering divergence can be improved effectively ; On the basis of centralized filter structure and federal filter structure, this paper designed a kinds of hybrid composite filter structure about multi-sensor system based on adaptive cubature kalman filter algorithm, and gave the method that fused the local filtering information of each sensor's to get the global filtering information; In the application background of positioning and navigation for vehicle to make simulation tests, and the results show the effectiveness of the proposed method.
\end{abstract}

\section{Introduction}

Kalman system under various filtering algorithm is one of the more widely technology used in multisensor data fusion processing. Standard Kalman filtering( Kalman Filter, KF) applies only to stochastic linear system, and mathematical model of the system can accurately; The filtered estimate for nonlinear systems, extend kalman filter(Extend Kalman Filter, EKF)is the most typical method, its first adopt the method of Taylor expansion and the second order truncation linearization of nonlinear system, and then continue to use the standard kalman filtering algorithm filtered estimate for the process of filtering, EKF need calculation of Jacobi matrix, computational complexity is high, and with a bigger truncation errors; No trace Kalman filtering (Unscented Kalman Filter, UKF) don't need to any form of approximation of nonlinear system, calculation of about a third of the EKF, filtering estimation accuracy can reach above three order Taylor expansion, but in the process of iterative filtering, the need for decomposition of matrices and inversion, the state estimation of covariance matrix is difficult to maintain is qualitative, in that case, the usability of the UKF are also affected, at the same time, the UKF Filter accuracy depends on the choice of parameters, the unreasonable parameters will cause large deviation of filtering. Volume Kalman filtering (Cubature Kalman Filter, CKF) is a newly proposed a nonlinear system determines sampling Filter method, the numerical integration method the volume criteria by recursive process approaching the a posteriori probability distribution, the optimal framework does not need to calculate jacobian matrix, also need not preset Filter parameters, thus received widespread attention, can be considered as a special case of the UKF, CKE restaurants did but CKF have more strict derivation, and more suitable for the filtering processing of high-dimensional nonlinear system.

CKF and standard KF, EKF, UKF filter algorithm, filtered estimate accuracy depends on accurate system model, the ideal condition. When the system model uncertainty and noise statistical properties of time-varying or deviating from the characteristics of gaussian white noise distribution, the accumulation of round-off error and measurement environment, great changes have taken place CKF filtering process will be severely affected, appear even filtering divergence. CKF is presented in this paper, on the basis of adaptive CKF is put forward, the method can according to the observation of system and history of filtering information constantly revised the filtering process, at the same time 
the system model parameters and noise statistical parameters estimation and correction, to form effectively restrain filtering divergence, in order to improve the filtering accuracy of estimation. Apply adaptive CKF to multi-sensor data fusion system, the design and the algorithm of hybrid composite filter structure, and realize the combination of multisensor data filtering and fusion.

\section{Adaptive kalman filter volume multisensor data fusion algorithm}

In the multi-sensor system, usually have different measuring characteristics between each sensor. Assume that independent among sensors, the measuring model of multi-sensor system can be expressed as follows:

$$
z_{k}^{i}=h_{k}^{i}\left(x_{k}, u_{k}\right)+v_{k}^{i}, i=1,2, \ldots, M
$$

In the formula, take the $i(i=1,2, \ldots, M)$ symbol corresponding to the parameters of the sensor $\mathrm{i}, v_{k}^{i}$ is the sensor i measurement noise, and there is $E\left[v_{k}^{i}\left(v_{j}^{i}\right)^{T}\right]=R_{k}^{i} \delta_{k j}, E\left[w_{k}\left(v_{j}^{i}\right)^{T}\right]=0$.

Boil down to, a combination of applied to multi-sensor data fusion filtering structure can be roughly divided into two categories: centralized and decentralized. Due to loss of filter the information before the less centralized filtering, thus has high filtering estimation precision, and fault-tolerant performance is poorer, high computational complexity is the main drawback of such a combination filter structure; Decentralized filter has a good stability and fault-tolerance, compared with the centralized filtering, its computation quantity is small, but the filtering precision is reduced. Put forward by Carlson federated filtering is one of the most widely used distributed filter method, its structure design, flexible filtering performance is good, can get through a simple and effective fusion times global optimal, the optimal state estimation, even by the attention. Federated filtering structure is presented in this paper, on the basis of reference and integrated the advantages of centralized filtering, this paper proposes a hybrid combination of filter structure. Each of these filter adopts adaptive CKF algorithm design. It can be seen that the structure of the composite filtering multiple information feedback process, and because of that, makes the filtering process of each filter correlation, and the related composite filter under the condition of difficulty in implementation. Carlson, variance upper bound technique is adopted, and based on the principle of information distribution effectively solve related under the condition of combination between the sub filter filter, principle of information distribution is refers to the whatever information distribution, the amount of information is unchanged

The amount of information $P^{-1}$ can be represented as state estimation information, Equation of state of information can be expressed as $Q^{-1}$. Information distribution is $P^{-1}$ and $Q^{-1}$ represent the amount of information the use of information distribution coefficient reasonably allocated between the main and sub filter that are $\left(P_{k \mid k}^{i}\right)^{-1}=\left(\lambda_{i}\left(P_{k \mid k}^{g}\right)^{-1}\right)^{-1},\left(Q_{k}^{i}\right)^{-1}=\left(\lambda_{i}\left(Q_{k}^{g}\right)^{-1}\right)^{-1} \quad(i=1,2, \ldots, M, m a)$, according to the principle of information distribution:

$$
\begin{gathered}
\left(P_{k \mid k}^{g}\right)^{-1}=\left(P_{k \mid k}^{m a}\right)^{-1}+\sum_{i=1}^{M}\left(P_{k \mid k}^{i}\right)^{-1} \\
=\left(\lambda_{m a}^{-1} P_{k \mid k}^{g}\right)^{-1}+\sum_{i=1}^{M}\left(\lambda_{i}^{-1} P_{k \mid k}^{g}\right)^{-1} \\
\left(Q_{k \mid k}^{g}\right)^{-1}=\left(Q_{k \mid k}^{m a}\right)^{-1}+\sum_{i=1}^{M}\left(Q_{k \mid k}^{i}\right)^{-1} \\
=\left(\lambda_{m a}^{-1} Q_{k \mid k}^{g}\right)^{-1}+\sum_{i=1}^{M}\left(\lambda_{i}^{-1} Q_{k \mid k}^{g}\right)^{-1}
\end{gathered}
$$

Which can be obtained:

$$
\lambda_{m a}+\sum_{i=1}^{M} \lambda_{i}=1
$$

Average distribution method is used to set distribution coefficient, namely:

$$
\lambda_{i}=(M+1)^{-1}, i=1,2, \ldots, M, m a
$$

There is $0<\lambda_{i} \leq 1$, and easy to get : $P_{k \mid k}^{g} \leq \lambda_{i}^{-1} P_{k \mid k}^{g}=P_{k \mid k}^{i}, Q_{k \mid k}^{g} \leq \lambda_{i}^{-1} Q_{k \mid k}^{g}=Q_{k \mid k}^{i}$ 。

In federal filtering process, the main and sub filter filter information by global filtering according to the corresponding information distribution coefficient reset, filtering the estimated error variance matrix and the system noise covariance matrix is intentionally amplified, and make $\lambda_{i}^{-1} P_{k \mid k}^{g}$ and $\lambda_{i}^{-1} Q_{k \mid k}^{g}$ 
into a diagonal matrix, repeatedly used in the process of recursive filtering the reset method, can remove the coupling relationship between the main and sub filter, this method corresponding is the basic principle of variance upper bound technique .

Based on the principle of information distribution, and USES the variance upper bound technique to adjust filtering process, the local estimate covariance matrix of each filter to get on the operation and process noise covariance matrices are related, that is $P_{k \mid k}^{i j}=0(i \neq j ; i, j=1,2, \ldots, M, m a)$, at this time easily according to the local state estimation for global integration formula of estimation. With the method of weighted combination, through the corresponding weights of each local state estimation error covariance inverse of normalization, and fusion shown by the following formula:

$$
\begin{gathered}
\hat{x}_{k \mid k}^{g}=P_{k \mid k}^{g}\left(P_{k \mid k}^{m a}\right)^{-1} \hat{x}_{k \mid k}^{m a}+P_{k \mid k}^{g} \sum_{i=1}^{M}\left(P_{k \mid k}^{i}\right)^{-1} \hat{x}_{k \mid k}^{i} \\
P_{k \mid k}^{g}=\left[\left(P_{k \mid k}^{m a}\right)^{-1}+\sum_{i=1}^{M}\left(P_{k \mid k}^{i}\right)^{-1}\right]^{-1}
\end{gathered}
$$

Get the global estimation $\hat{x}_{k \mid k}^{g}$ is optimal unbiased, and can prove that: $P_{k \mid k}^{g} \leq P_{k \mid k}^{i} \quad(i=1,2, \ldots, M, m a)$, the global optimal estimation is better than that of the local estimates. Like on type, local information system is given based on the formula of noise statistical characteristics of fusion, as follows:

$$
\begin{gathered}
q_{k \mid k}^{g}=Q_{k \mid k}^{g} \sum_{i=1}^{M}\left(Q_{k \mid k}^{i}\right)^{-1} q_{k \mid k}^{i} \\
Q_{k \mid k}^{g}=\left[\sum_{i=1}^{M}\left(Q_{k \mid k}^{i}\right)^{-1}\right]^{-1}
\end{gathered}
$$

Among them, $q_{k \mid k}^{i}$ and $Q_{k \mid k}^{i}$ is made by the c is the filter system noise statistical properties in time revised.

\section{The application and simulation analysis}

Of vehicle navigation and positioning generally adopts the GPS/DR integrated navigation system, because of the U.S. military GPS control, thus in military applications should try to reduce reliance on GPS. Beidou navigation positioning system (BDS) is now in the perfect stage, basic positioning navigation function has been covered throughout our country, but the positioning precision is not ideal, based on this, using GPS/BDS/DR navigation system, and by using multi-sensor combination filtering method based on adaptive CKF navigation of the three kinds of sensor data filtering and fusion, in order to improve the navigation and positioning accuracy.

If not considering the elevation changes, the movement of the vehicle can be treated as a two-dimensional plane movement, fixed point as the origin of coordinates, and the east and north direction in order to establish the geodetic coordinate system axis, the kinematics of the vehicle state vector option is: $x=\left[s_{e}, \vartheta_{e}, a_{e}, s_{n}, \vartheta_{n}, a_{n}\right]^{T}$,for each component, the corresponding Yu Zhengdong and due north direction, speed and acceleration, the coordinates of the unit are: $\mathrm{m}, \mathrm{m} / \mathrm{s}, \mathrm{m} / \mathrm{s}^{2}, \mathrm{~m}, \mathrm{~m} / \mathrm{s}, \mathrm{m} / \mathrm{s}^{2}$; According to Newton's law and the current statistical model, a sampling time interval for, will be after discretization of vehicle motion model is as follows: $x_{k+1}=f_{k+1}\left(x_{k}, u_{k}\right)+w_{k}=F_{k+1} x_{k}+u_{k}+w_{k}$.

The simulation experiments, the assumption is that the vehicle in a certain period of the road with a turn for the random acceleration movement. Starting point of turning the initial moment vehicles ahead 150 meters; Turning part is the radius of 100 meters, a circular radian is 90 degrees, it make the road from the northeast to the southeast direction; 150 metres before turning vehicles do uniformly retarded motion, turning process for uniform circular motion, turning after a period of time, you do uniformly accelerated motion.

The simulation time is $t=100 \mathrm{~s}$, sampling time interval is $T=1 \mathrm{~s}$; The initial filter state estimation is $\hat{x}_{0 \mid 0}=[0.8,10,0.9,16,13,1.1]^{T}$; Error covariance matrix is $P_{0 \mid 0}=\operatorname{diag}[20,5,2,21,5.2,2]^{T}$; Each sensor measurement noise and the system noise of the initial mean to 0; System initial covariance matrix is $Q_{0}=[30,5,2,35,8,3]^{T} ;$ GPS, BDS and DR initialized to measurement noise covariance matrices $R_{0}^{G P S}=R_{0}^{B D S}=[30,10,35,12]^{T}, R_{0}^{D R}=\left[10,(2 \pi / 180)^{2}\right]^{T}$; Information distribution coefficient $\lambda=0.25$; To facilitate comparative analysis, experiment and combination of multiple sensors based on EKF 
filter method, the simulation experiment applied EKF filtering, assuming that the statistical characteristic of noise after initialization is unchanged.

The simulation results show that the information provided by each sensor in multi-sensor system can be fused, which can make the information more accurately describe and estimate the state of the monitored object, the premise is that the fusion method is reasonable. Adaptive CKF compared to EKF the classic nonlinear filtering method is more applicable in filtering system model, the measurement equation is not accurate, have good inhibitory effect on filtering divergence; based on adaptive CKF multi-sensor system combined filtering method can effectively with the sensor information fusion, improve the accuracy of the information; in addition, compared to the filtering process of the adaptive CKF EKF is more simple and computational efficiency higher, easier engineering realization and application.

\section{Conclusion}

To join a group of noise statistics estimator and the modified function of cubature Kalman filter algorithm is improved by the proposed adaptive cubature Kalman filter algorithm; for the filtering problem of the combination of multi sensor system was studied based on adaptive cubature Kalman filtering multi-sensor system combined filtering structure is presented, and based on cubature Kalman filter for multi sensors data fusion method; simulation experiment results show that the method presented in this paper to restrain the divergence of Kalman filter and improve the estimation precision, and multi sensor system combination filter has good effect. The adaptive cubature Kalman filter algorithm assumes that the ideal conditions of normal distribution of the statistic characteristic of the speckle noise compliance, so under the other condition is worse, this method may not applicable; in addition, for each sensor in the multi-sensor system under the condition of correlated combination of filter paper is also not yet involved, in the future work will be this in-depth study.

\section{References}

[1] JULIER S J, UHLMANN J K.A new method for the nonlinear transformation of means and covariances in filters and estimators [J].IEEE Trans on Automatic Control, 2000, 45 (3):477-482.

[2] ARASARATNAM I, HAYKIN S. Cubature Kalman Filters [J]. IEEE Trans. On Automatic Control, 2009, 54 (6):1254-1269.

[3] D.LOEBIS, R.SUTTON,J. CHUDLEY. .Adaptive tuning of a Kalman filter via fuzzy logic for an intelligent AUV navigation system [J].Control Engineering Practice,2004, 12(12) 1531-1539.

[4] T. D. POWELL. Automated Tuning of an Extended Kalman Filter Using the Downhill Simplex Algorithm [J].Journal of guidance, control and dynamics. 2002, 25(5): 901-908. 